\title{
REVITALIZACIÓN PARCIAL DEL HOSPITAL DE LAS CINCO LLAGAS DE SEVILLA COMO SEDE DEL PARLAMENTO DE ANDALUCÍA
}

\author{
(PARTIAL REVITALIZATION OF THE CINCO LLAGAS HOSPITAL IN SEVILLA AS \\ THE SEAT OF THE PARLIAMENT OF ANDALUCIA)
}

\author{
Pedro Rodríguez Pérez, arquitecto
Miembro de la Academia del Partal (Asociación Libre de Profesionales de la \\ Pedro Rodríguez Pérez, arquitecto
Miembro de la Academia del Partal (Asociación Libre de Profesionales de la \\ Restauración Monumental)
}

ESPAǸA

Fecha de recepción: 15 - II - 94

\section{RESUMEN}

El Hospital de las Cinco Llagas de Sevilla fue trazado por Martin de Gainza a mediados del siglo XVI, disponiendo las salas de enfermos en doble crucero y rodeándolas por patios cuyas naves perimetrales exteriores completan una gran planta rectangular.

La iglesia, creada por Hemán Ruiz el Mozo, se sitúa en el patio principal, dominando con su gran altura y su severo volumen la masa horizontal del conjunto, soberbio telón de fondo de la explanada que lo separa de la muralla almohade de la ciudad.

El notable edificio, que ha conservado su función sanitaria hasta hace poco más de nina década, es desde el veintiocho de febrero de 1992 la sede del Parlamento de Andalucia.

Este articulo es un apretado resumen de la historia del viejo Hospital, de su estado a principio de los años ochenta y de los estudios, proyectos y obras llevadas a cabo sobre él para restaurarlo y adaptarlo a los muevos usos.

\section{SUMMARY}

The Hospital of the Cinco Llagas of Sevilla was designed by Martin Gainza in the middle of XVI century. It had patient's rooms disposed in the form of a double cross surrounded by patios with exterior perimetric aisles making up a large rectangular floor.

The church, created by Heman Ruiz el Mozo, is located in the principal patio and, with its height and its grim volume, it dominates the horizontal mass of the whole, representing a magnificent background for the esplanade which separates it from the Almohad city walls.

This remarkable building which had preserved its medical function until a little longer than a decade ago, has been the seat of the Parliament of Andalucia since 28 February, 1992.

This article is a brief summary of the history of the Hospital, its condition at the beginning of the eighties and of the studies, projects and the works carried out on the building in order to restore it and adapt it to the neu' use.
El Hospital de las Cinco Llagas o de la Sangre está situado a unos doscientos metros al Norte de la muralla almohade de Sevilla, junto al antiguo Camino Real que penetraba en la ciudad por la vecina Puerta de la Macarena.

Originalmente fue la segunda sede de la fundación de caridad que, para atender mujeres enfermas, creara $D^{2}$. Catalina de Ribera a principios del siglo XVI, y su uso hospitalario, ampliado a los varones, se ha conservado desde su inauguración en 1559 hasta los años setenta de nuestro siglo.

Su visión exterior impone, pues se trata de una noble construcción de dos pisos de gran altura, de fábrica pétrea modulada por pilastras y semicolumnas, cuyo volumen, de haberse labrado en su totalidad, habría sido un gran prisma torreado en sus cuatro esquinas, de ciento setenta y tres por ciento cincuenta y seis metros de base, orientado según los puntos cardinales, del que emerge el volumen cruciforme de su iglesia. 


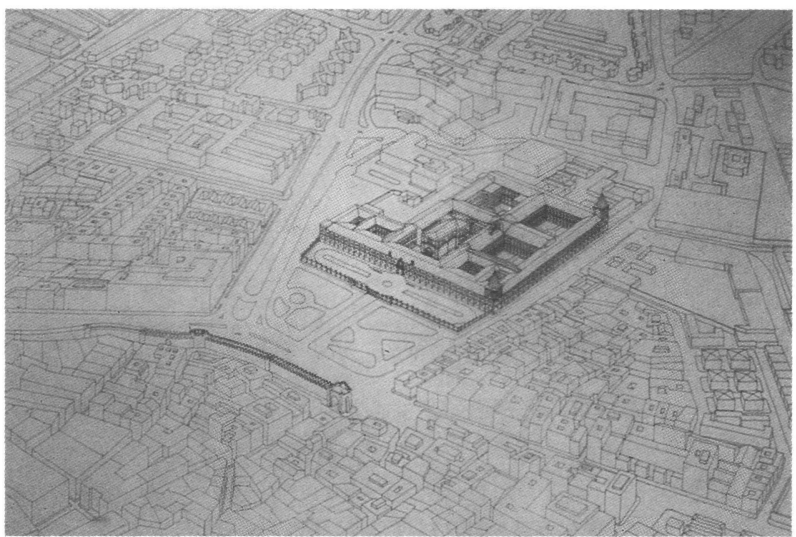

El edificio del Hospital de las Cinco Llagas en la Ciudad. Dibujo de alumnos de la ETSAS bajo la dirección del profesor Martinez Zúniga. (Foto: A. Jiménez).

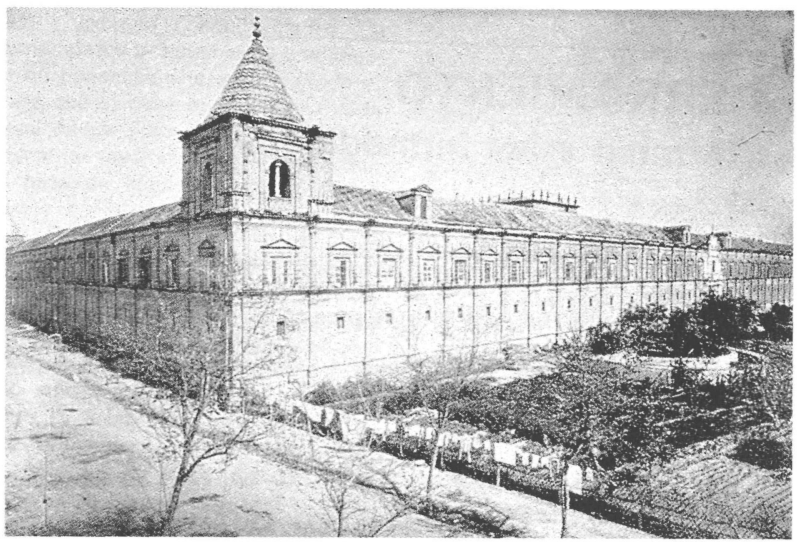

Esquina Suroeste del Hospital hacia 1880. Foto de la reedición del Album del Vizconde Vigier (extraída de la Memoria del Estudio Preliminar sobre el Hospital de las Cinco Llagas. A. Jiménez y P. Rodríguez).

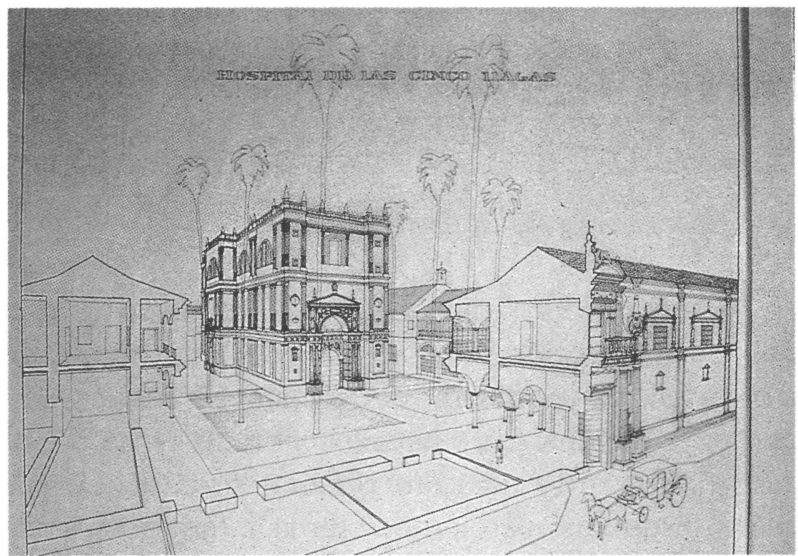

Perspectiva seccionada del Hospital mostrando la iglesia en el Patio del Recibimiento. Dibujo de alumnos de la ETSAS bajo la dirección del profesor Martinez Zúñiga. (Foto: A. Jiménez).

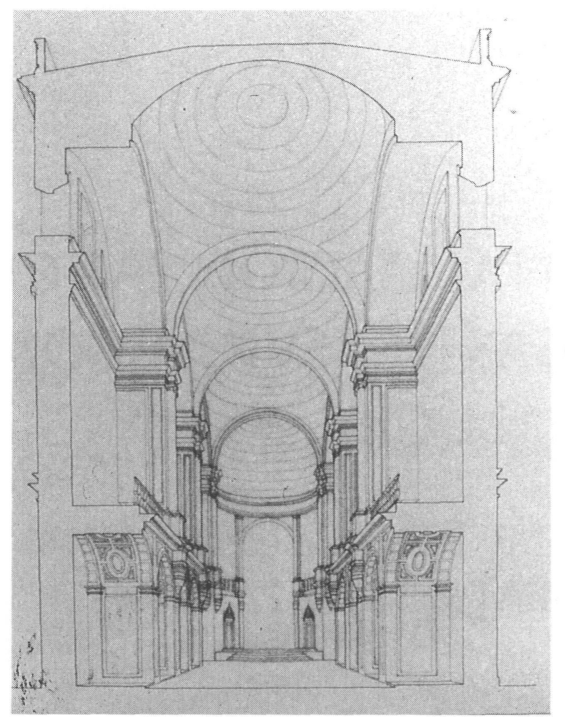

La iglesia de Hernán Ruiz. Perspectiva interior. Dibujo de alumnos de la ETSAS bajo la dirección del profesor Martinez Zúniga. (Foto. A. Jiménez).

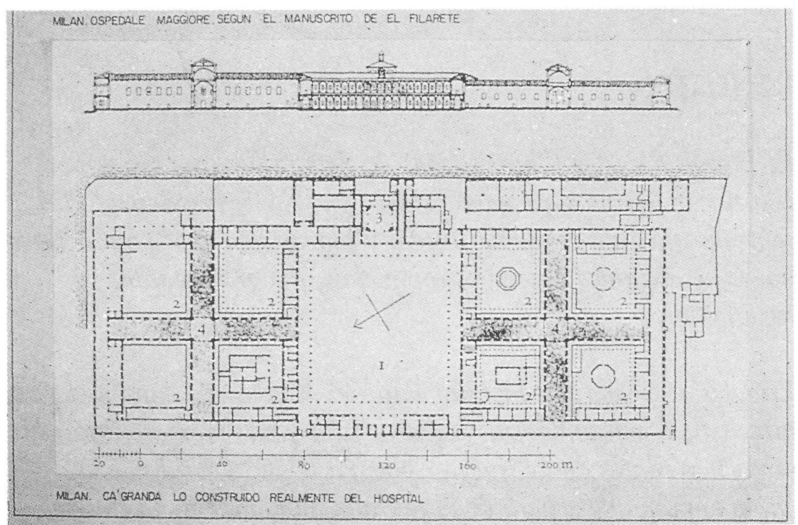

Milán. Sección y planta real del Ospedale Maggiore. Diseño del Filarete. (Extraídas de la Memoria del Estudio Preliminar sobre el Hospital de las Cinco Llagas. Estudio de A. Jiménez y P. Rodriguez).

Su fachada meridional, que mira a Sevilla, contiene la puerta principal, que da acceso al patio del Recibimiento, en el que se yergue la excepcional capilla, debida al genio de Hernán Ruíz el Mozo, segundo de los varios Maestros Mayores que en el edificio trabajaron e inmediato sucesor del que fue su tracista y primer director de sus obras, Martín de Gaínza.

Tanto la singular disposición de la iglesia, exenta en el patio central, como la organización en doble crucero de las naves de enfermos, han dado lugar a que se proponga como modelo de su traza el Hopedale Maggiore de Milán, diseñado por el Filarete. 


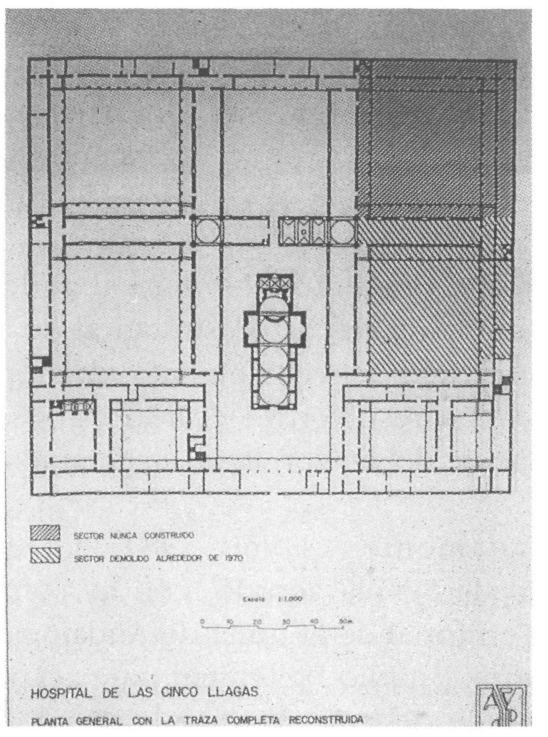

Sevilla. Hospital de las Cinco Llagas. Inducción de la traza completa y zonas no acabadas o demolidas, según J.M. Jiménez y $P$. Rodriguez. (Dibujo: Estudio A.J. y P.R.; foto: P. Rodríguez).

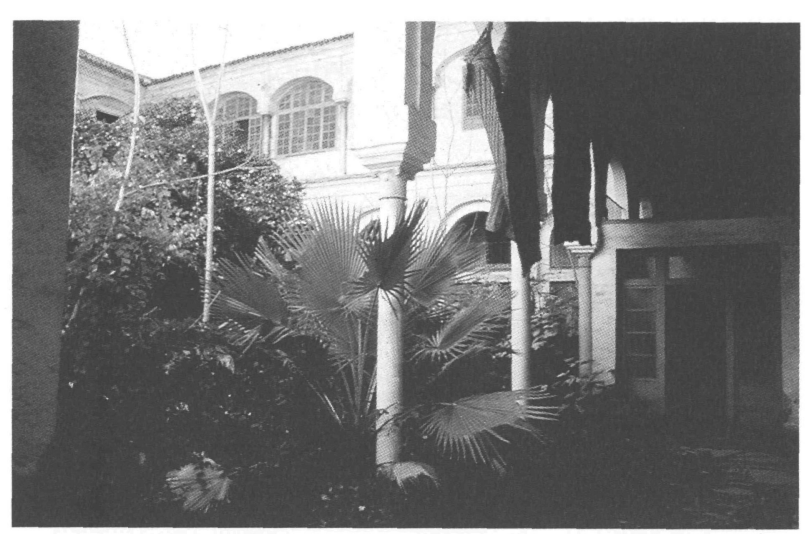

El Hospital a finales de los ochenta. Patio de S. Carlos (foto: P. Rodriguez, 1987).

Puede decirse que hacia finales del siglo XVII se acaban las intervenciones edilicias de cierto alcance en el Hospital, hasta llegar a nuestros días, en que las "modernas exigencias" sanitarias y el carácter de Hospital Universitario que adquirió Cinco Llagas lo llevaron a soportar fuertes transformaciones, sin importar para nada la percepción de la unidad formal del conjunto ni su integridad constructiva. A pesar de tales agresiones, la circunstancia de ser el Hospital General de Sevilla, lo preservó de la ruina.

Tras el terremoto de 1968, los responsables de conservarlo decidieron su demolición parcial, que se llevó por delante, entre otras, la más oriental de las naves de enfermos mientras que éstos y el personal

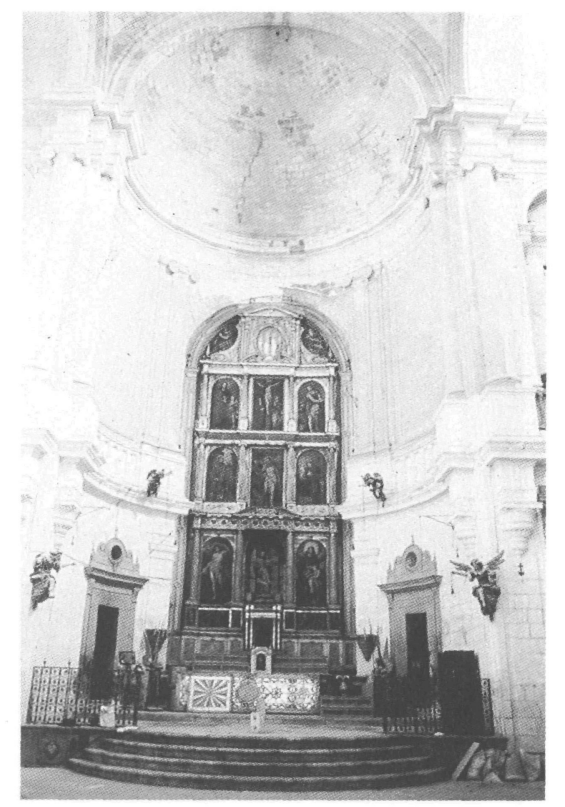

El Hospital a principios de los ochenta. Interior de la iglesia hacia el preshiterio. (Foto: P. Rodriguez. 1983).

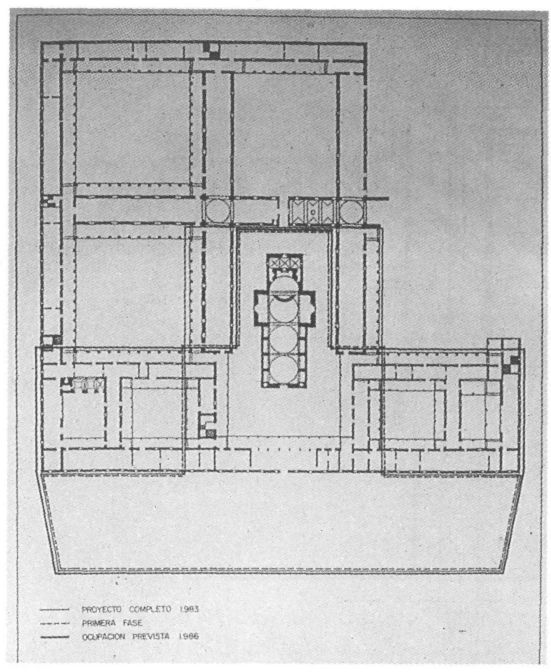

Planta baja general del Hospital y fases de actuación, con sus ámbitos respectivos. (Dibujo: Estudio A.J. y P.R.; foto: P. Rodriguez).

sanitario siguieron conviviendo con los apuntalamientos en el resto de las dependencias, hasta que al principio de los años ochenta se desalojaron las últimas, entonces ocupadas por los Servicios Farmacéuticos de la Diputación Provincial sevillana, que era la dueña del monumento.

Esta década larga de descuido aceleró el deterioro de la maltrecha fábrica, en la que se arruinaron tejados, se agrietaron muros y se volcaron arquerías, víctimas de los empujes que las estructuras de cubierta y los alfarges de sus pisos producían al perder sección y desorganizarse sus entregas por la acción de toda suerte de organismos xilófagos. 


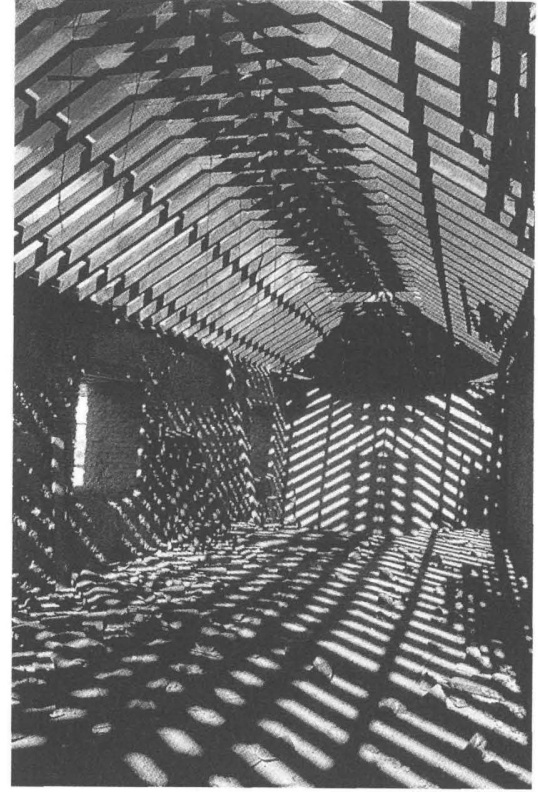

Cubierta de par y nudillo de una nave menor, durante las obras de revitalización. (Foto: P. Rodriguez, 1990).

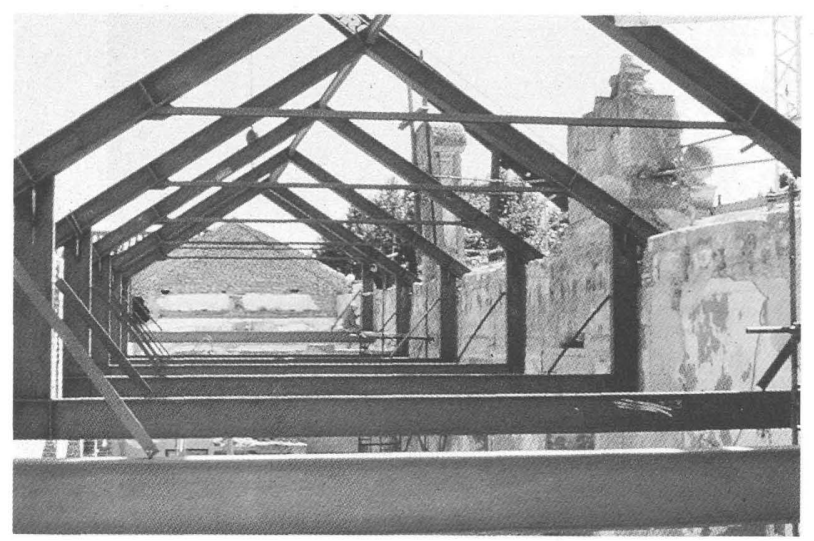

Nuevas estructuras bajo cubiertas de las crujias de fachada para alojar servicios. (Foto: M.L. Marín, 1983).

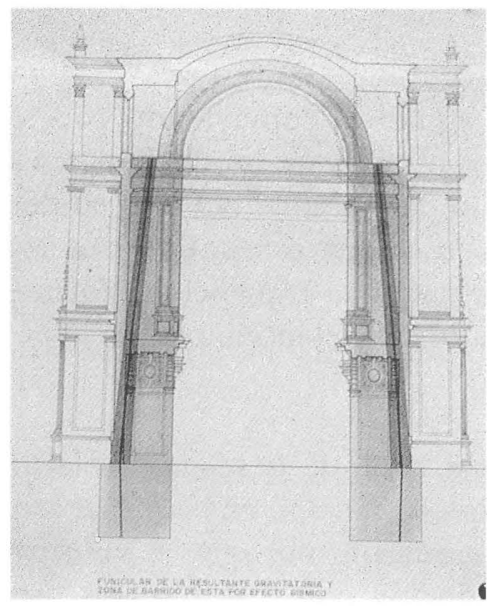

Sección transversal de la iglesia mostrando las trayectorias de los esfuerzos a lo largo de los machones centrales de la nave principal, bajo cargas permanentes y sismo, según E. Machuca. (Foto: P. Rodríguez, 1986)
Los muros, empapados de agua, vieron crecer frondosas higueras en sus grietas, y los patios se vieron invadidos por la maleza que, al secarse en verano, aumentaba el ya alto riesgo de incendios del conjunto.

En la iglesia, por entonces muy abandonada, se hicieron obras de refuerzo estructural de sus bóvedas y muros que, inevitablemente, dejaron muchos problemas sin resolver. El estado de los retablos, especialmente del mayor, llegó a ser lamentable.

Afortunadamente, el año 1981 la Diputación Provincial de Sevilla acordó, con la Consejería de Política Territorial de la Junta de Andalucía, la realización de un Estudio Preliminar para aclarar los términos en que debería moverse la recuperación del edificio.

Dicho estudio, abarcó múltiples aspectos, dando lugar a una serie de conclusiones y propuestas de intervención que se han respetado en los subsiguientes proyectos.

Durante 1982 se realizaron unas obras urgentes de

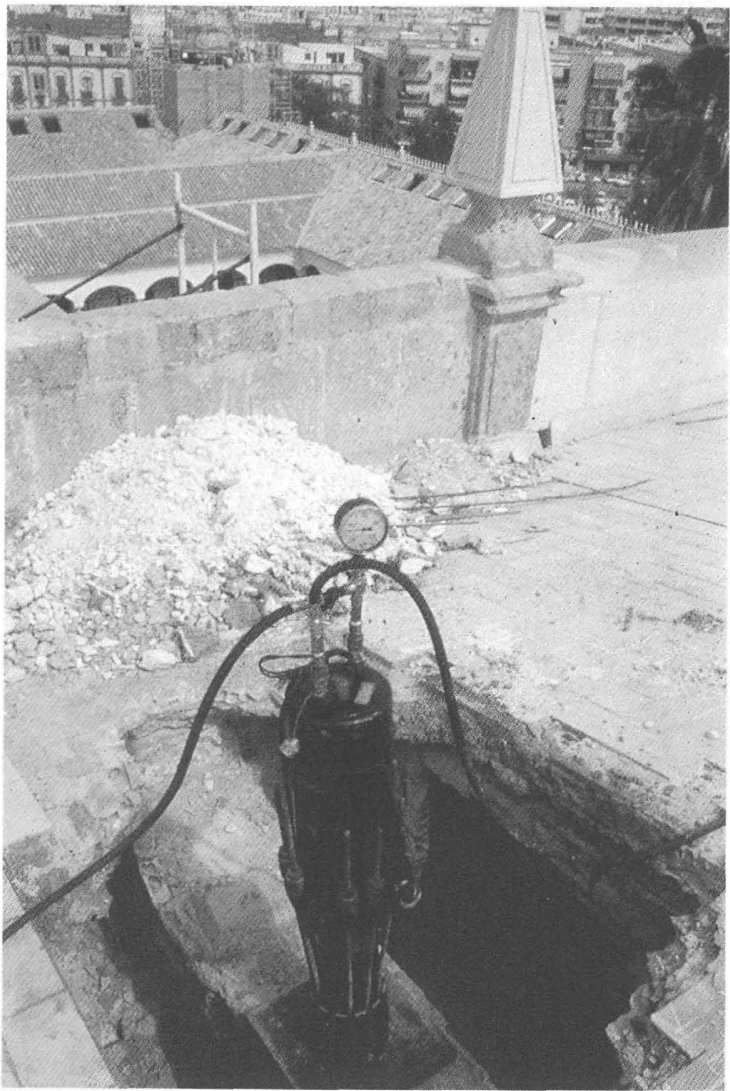

Gato para la operación de tesado de cables dispuestos en los machones de la ilustración anterior. (Foto: P. Rodríguez, 1991). 
consolidación del tercio central de la crujía de entrada y la galería adyacente, sufragadas por la Diputación Provincial, que acabó cediendo el Hospital a la Junta de Andalucía, quien lo adscribió inicialmente al Parlamento Autonómico, para que éste dispusiera en él su tercera y definitiva sede.

Desde noviembre de ese mismo año, el Parlamento de Andalucía ha gestionado, prácticamente en solitario, todo el proceso de proyección arquitectónica y las obras de la mitad sur del enorme inmueble.

El primer proyecto completo de revitalización se concluyó el trece de septiembre de 1983, y hasta el cinco de mayo de 1987 no se comenzaron las obras de la Sede Parlamentaria, cuya inauguración tuvo lugar casi cinco años más tarde, el 28 de febrero de 1992.

Estas obras han perseguido los siguientes objetivos:

- Consolidar íntegramente las construcciones antiguas, pertenecientes a la configuración original del monumento.

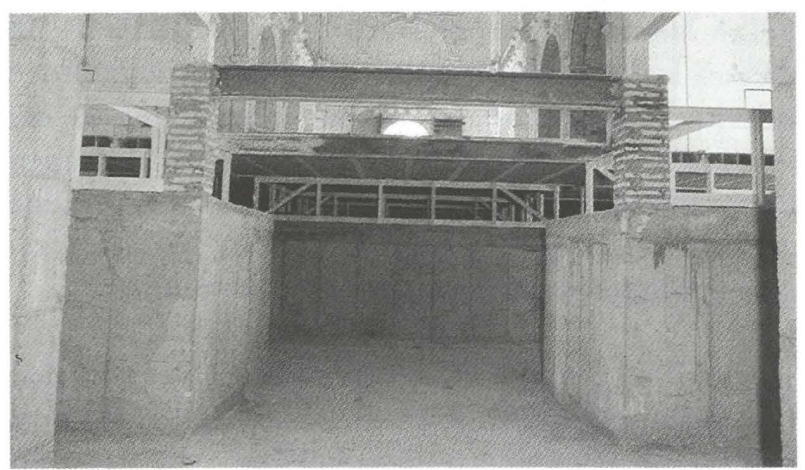

El subsuelo técnico del Salón de Plenos en la antigua iglesia. (Foto: TECNIFOTO).

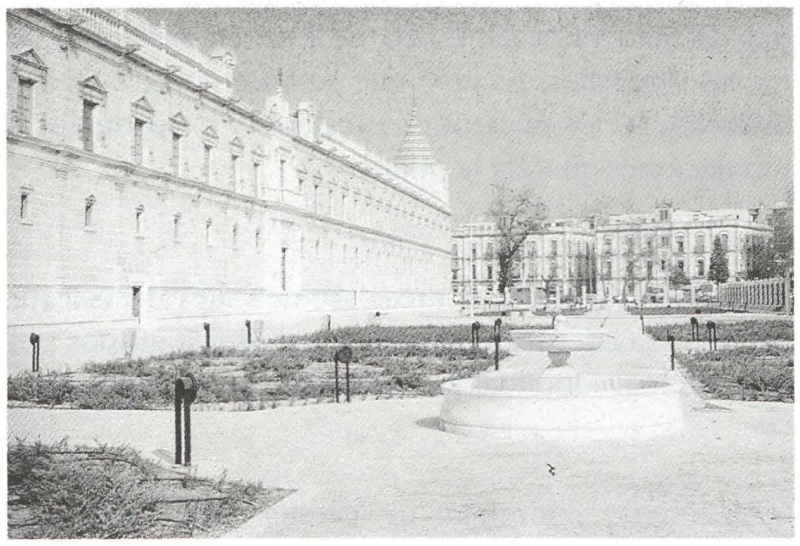

La fachada y los jardines meridionales del Hospital, tras la intervención. (Foto: TECNIFOTO).
Con este fin, se han recalzado sus cimentaciones empleando diversas técnicas, desde las tradicionales hasta la inyección o el micropilotaje. Se han saneado y reforzado sus muros, aplomado sus arquerías y relevado estructuralmente sus artesonados (restaurándolos o sustituyéndolos, según su estado), con forjados metálicos dispuestos en el espacio que ocuparon las primitivas alcatifas.

Las cubiertas de tejas se han renovado por completo, así como sus estructuras, que ahora son forjados inclinados sobre pórticos triangulares o pentagonales biapoyados.

La iglesia se ha reforzado con tirantes metálicos pasivos, libres por encima de las cornisas de arranque de sus bóvedas o adheridos a su fábrica en los machones de esquina de su crucero, mientras que los grandes pilares intermedios de los últimos tramos de su nave principal se han provisto de anclajes verticales postensados para centrar la acción combinada del arco transversal y los esfuerzos sísmicos, micropilotándolos para evitar asientos complementarios. Sus grietas han sido grapadas y se han reforzado los bordes de sus ventanales con la propia carpintería metálica diseñada al efecto.

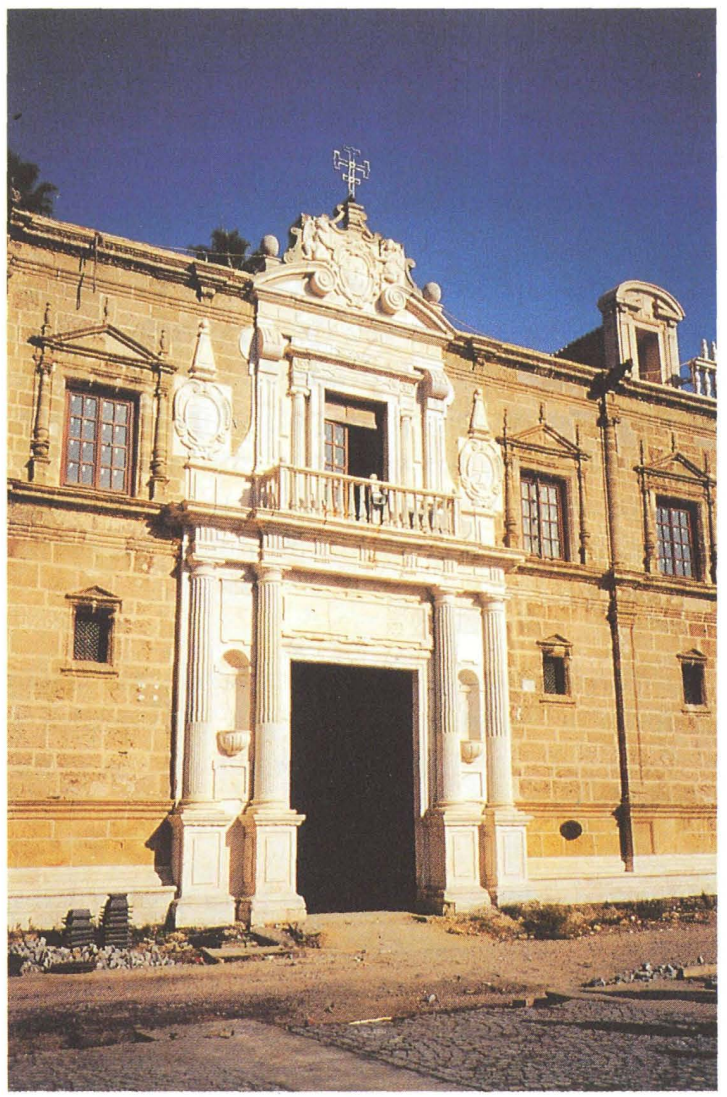

La portada principal, debida a Hernán Ruíz y Asensio de Maeda, al final de las obras. (Foto: P. Rodriguez, 1991). 


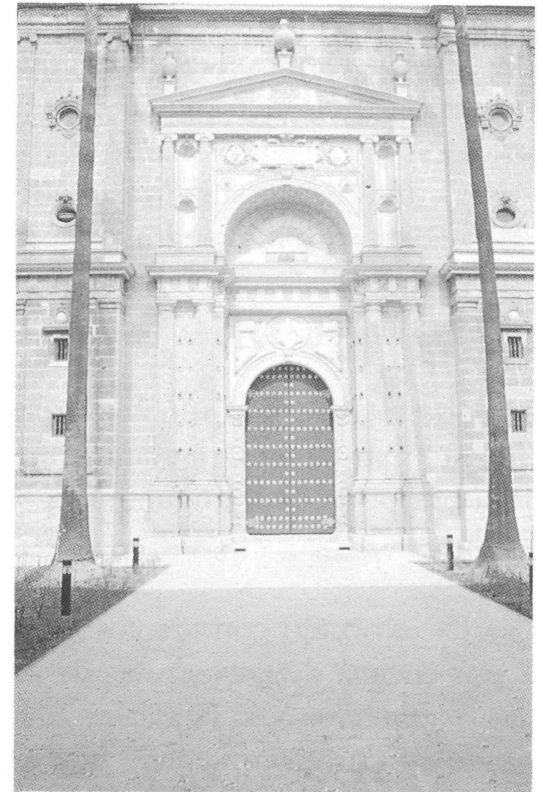

La portada Sur de la antigua iglesia, diseñada por H. Ruiz y con esculturas de B. Vázquez el Viejo, tras la restauración ejecutada por los profesores J. Abad y S. Martínez. (Foto: TECNIFOTO, 1992).

- Devolver su carácter a los patios y galerías, así como a los espacios interiores principales.

Con este empeño, se ha eliminado la construcción y la vegetación parásita para reponer la jardinería adecuada en los primeros, se han liberado las galerías de todas sus particiones y cierres opacos y de sus entreplantas, que sólo se han reeditado en las naves cerradas de menor latitud.

En la antigua iglesia, convertida en Salón de Sesiones Plenarias una vez desacralizada, se ha limpiado, consolidado e hidrofugado la sillería, se ha restaurado su retablo principal, retirando todos los demás, así como los diversos enterramientos, en cuidadosa coordinación con las Autoridades Eclesiásticas, Sanitarias y Mortuorias, y los particulares afectados.

Se ha renovado la balaustrada de su triforio, siguiendo las pautas dejadas por su creador y se han despejado los huecos laterales de sus ventanales, de tipo termal.

El indispensable equipamiento para acoger los nuevos usos, se ha concebido con un carácter eminentemente mobiliar. Incluso el Escudo de Andalucía, que preside los actos políticos ocultando ordinariamente el retablo mayor, se ha hecho abatible para permitir la contemplación de éste durante los períodos de visita establecidos.

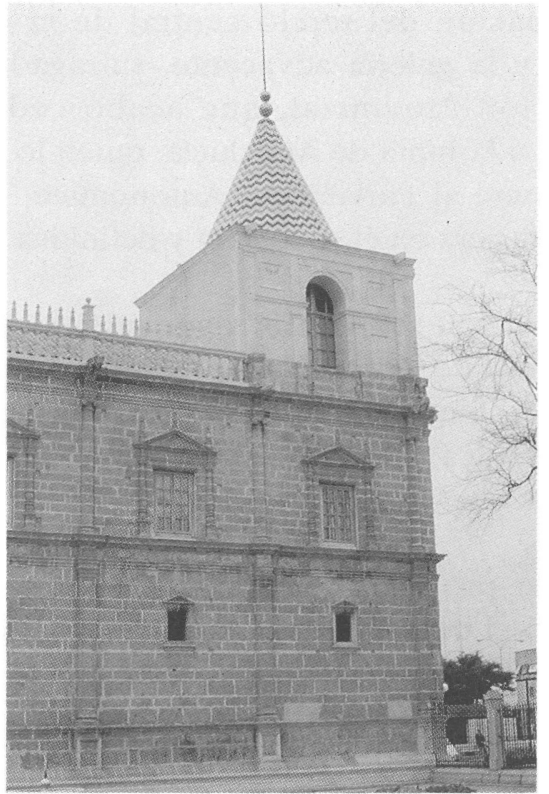

La esquina Sureste del Hospital mostrando el nuevo remate de la torre. (Foto: G. Pavón, 1992).

- Dotar los espacios de trabajo de la mayor flexibilidad posible.

Así, a las naves de enfermos de planta baja incluidas en la zona parlamentaria, se les ha devuelto su primitiva diafanidad, y las funciones a que se han destinado (Biblioteca y Salón de Usos Múltiples) han permitido dotarlas de un mobiliario permanente de cierto empaque, en el primer caso, o adaptarlo a las necesidades del momento en el segundo.

Las correspondientes de planta alta se han tenido que dividir para albergar las cuatro Salas de Comisiones exigidas y unos locutorios para radiofonía, si bien las particiones aparentan un carácter mobiliar al estar revestidas de madera, a diferencia de los paramentos de los muros originales, recurso que se ha empleado para la inserción de los núcleos de escaleras y los patinillos de instalaciones en toda la obra.

Las naves dedicadas a tareas administrativas institucionales o a las dependencias de los distintos grupos políticos, se han subdividido con mamparas removibles.

- Ordenar y completar el sistema circulatorio y de accesos del conjunto adaptándolo a las exigencias de confort y de seguridad actuales. 


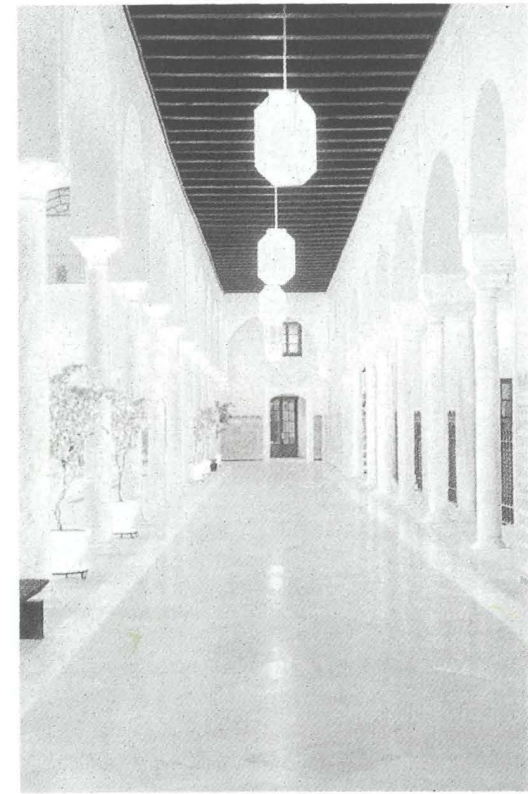

La galería Sur del Patio del Recibimiento tras las obras. (Foto: TECNIFOTO, 1992).

Se han dispuesto con esta finalidad once nuevos núcleos de escaleras y ascensores (accesibles a minusválidos en sillas de ruedas) que conectan las plantas originales del inmueble con los desvanes, entreplantas y sótanos de nueva creación. Además se han instalado varios montacargas, y una gran plataforma elevadora une el Salón de Usos Múltiples con el sótano inmediato.

Las conexiones con el exterior se han mejorado, añadiendo a las cuatro puertas preexistentes tres más para peatones y dos para vehículos.

- Dotar al edificio de las instalaciones adecuadas para su correcto funcionamiento como sede del Legislativo Andaluz.

Esto nos ha obligado a la concepción y ejecución de toda una serie de espacios auxiliares, la mayoría de ellos subterráneos, aunque otros muchos se han dispuesto bajo las cubiertas, que enlazados convenientemente mediante galerías, técnicas y patinillos verticales, alojan toda la maquinaria y las conducciones, sirviéndose desde allí y por falsos techos, los espacios de trabajo, facilitando sus eventuales remodelaciones y reduciendo las molestias de su mantenimiento.

De las múltiples instalaciones dispuestas, destacaremos sólo los extremos que nos parecen de mayor interés.

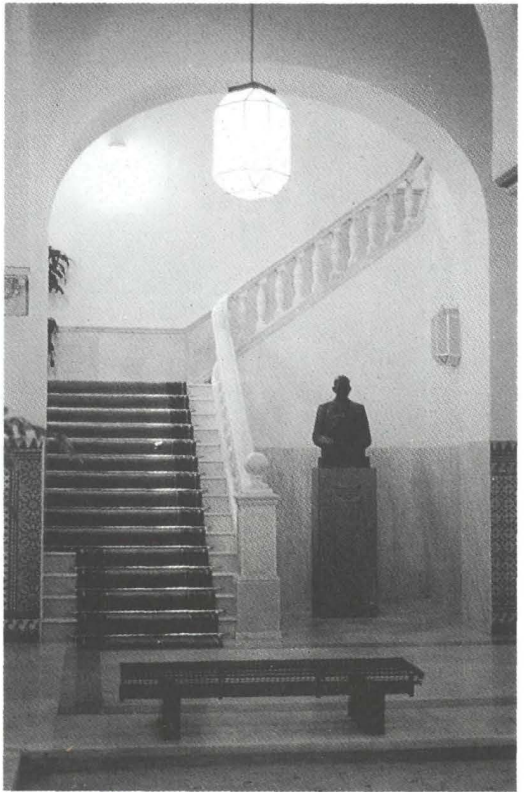

Escalera principal con el busto de Blas Infante. (Foto: G. Pavón, 1992).

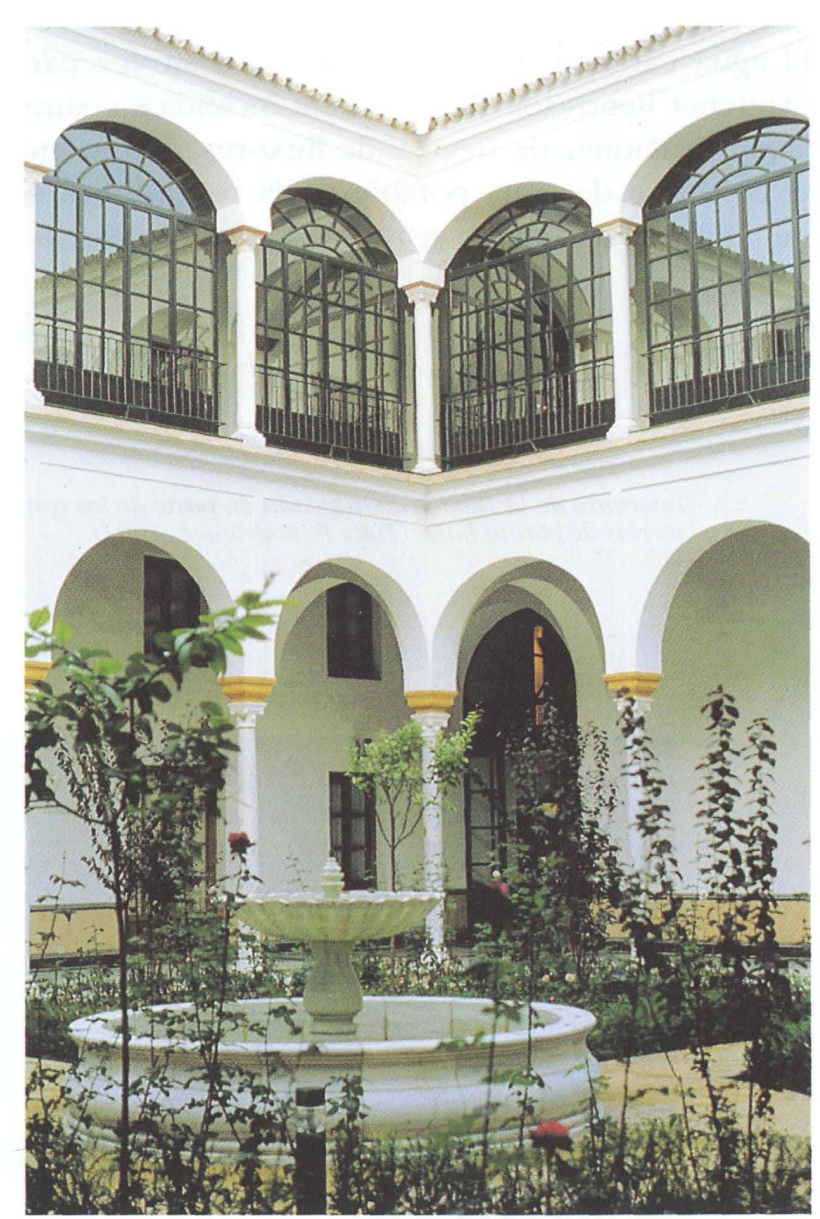

Vista del rincón Sureste del Patio del Alcohol, una vez restaurado (Foto: G. Pavón, 1992). 


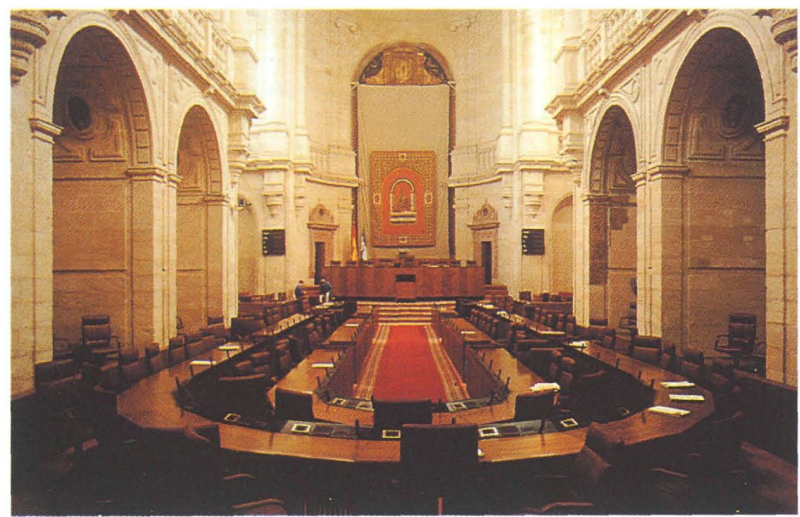

Interiores del Salón de Plenos. (Foto: P. Rodríguez, 1992)

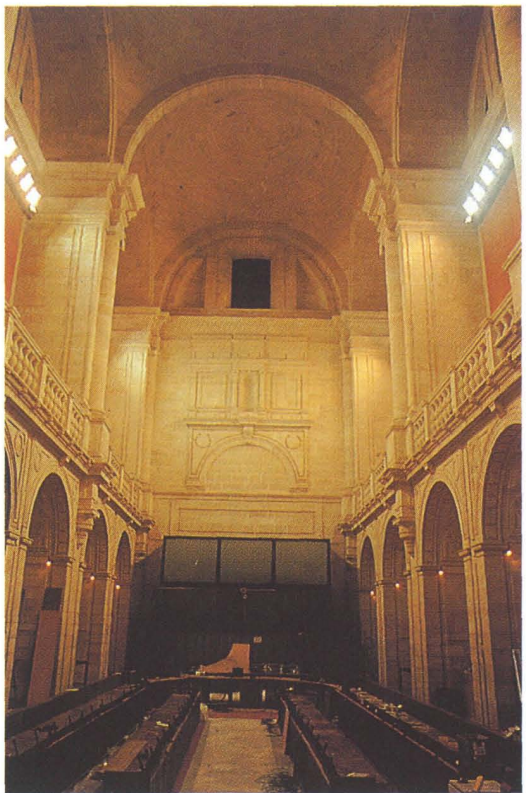

El acondicionamiento de aire, por ejemplo, utiliza como fuente térmica el agua subterránea, lo que garantiza todo el año un elevado rendimiento energético y una contaminación prácticamente nula.

El agua extraída del terreno se usa a su vez para mantener llenos los aljibes contra incendios y surtir las instalaciones de riego y de fluxores, con lo que el consumo de agua potable de la red pública se minimiza. El excedente se reinyecta en el subsuelo.
El Salón de Plenos se ha iluminado de forma que las sesiones se puedan grabar en televisión a color, para lo que se han instalado cámaras telemandadas desde una central dotada de los instrumentos necesarios para la completa realización de programas, con independencia de las tomas que puedan hacerse por emisoras autorizadas.

Su megafonía interior se ha cuidado especialmente para resolver el problema de reverberación del recinto,
Interiores de la Biblioteca, ubicada en parte de las antiguas enfermerias de planta baja. (Foto: P. Rodríguez, 1993).

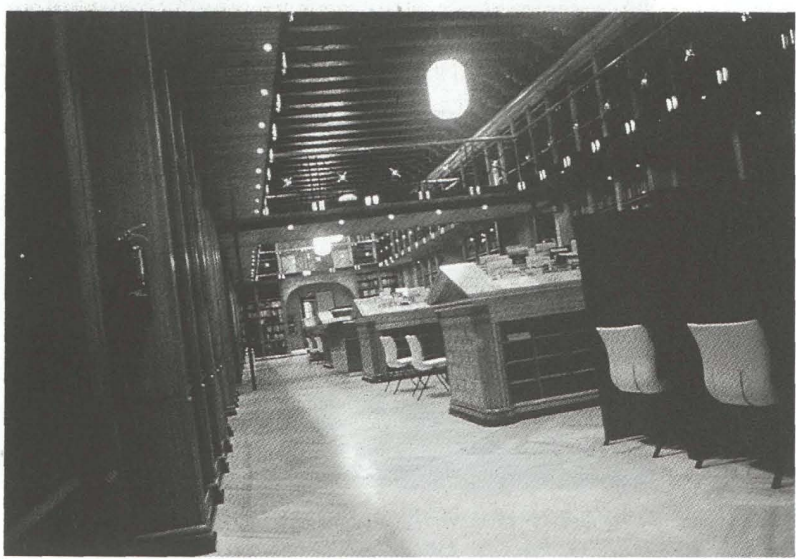

(c) Consejo Superior de Investigaciones Científicas Licencia Creative Commons 3.0 España (by-nc)

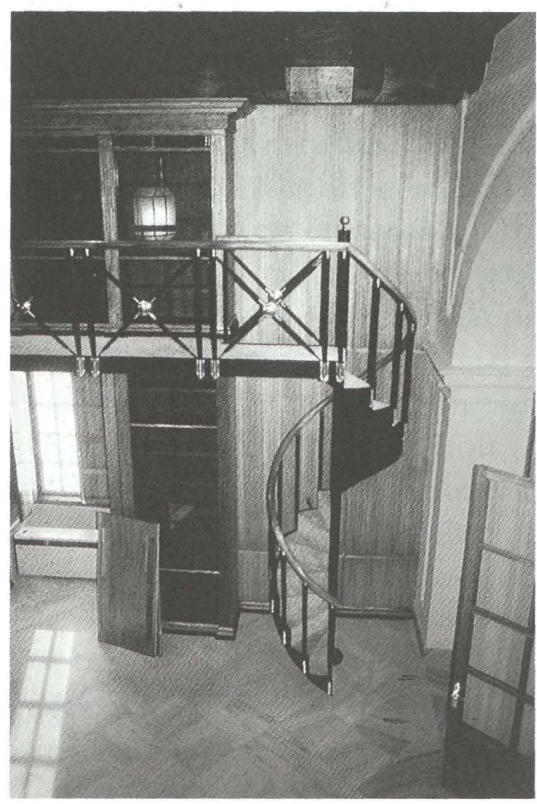

http://informesdelaconstruccion.revistas.csic.es 
paliado previamente con acondicionadores acústicos que ocupan los lugares antes destinados a los retablos en las capillas laterales, así como los canceles de nueva creación, sirviendo al mismo tiempo para ocultar los enormes conductos de impulsión del aire acondicionado.

Se han instalado los dispositivos de seguridad que una institución de estas características conlleva, y todas las dependencias están comunicadas por la red integrada de voz y datos, hoy inevitable en cualquier edificio administrativo.

El conjunto de las instalaciones se gobierna desde un Centro de Control Automático lo que, se supone, facilitará la explotación del edificio.

- Recuperar la imagen pública del Hospital adaptándola al nuevo entorno urbano.

Con esta voluntad se han restaurado sus fachadas reponiendo parcialmente la balaustrada que tuvieron como remate, se ha aplacado con piedra el tramo más meridional de su cara este, antes simplemente encalada, cuando daba a unas antiguas huertas y se ha completado la torre sureste, sintetizando en su composición los rasgos formales de las dos preexistentes.

Por último, se han despejado los espacios exteriores propios del edificio de su moderna arboleda, sustituyéndola por jardines geométricos de setos y macizos de flores, y se ha solicitado al Ayuntamiento de Sevilla que haga lo propio con los jardines públicos delanteros que ocultan inaceptablemente el edificio por todo su frente sur.

Tras todas estas obras, nuestro Parlamento se ha instalado en las naves y galerías que rodean los cinco patios meridionales del viejo hospital y que, de este a oeste, son los siguientes:

- Patios del Cobalto y del Alcohol, destinados básicamente a funciones administrativas de la Cámara, aunque una pequeña parte la ocupa el Consejo Asesor de R.T.V. en Andalucía.

- Patio del Recibimiento, cuyos espacios más significativos son el Salón de Plenos, las Salas de Comisiones, la Biblioteca, la Sala de Usos Múltiples y la Presidencia de la Cámara, además de la cafetería y los grandes archivos.

- Patios de San Carlos y de la Botica, cuyas dependencias se ocupan por los despachos de los demás Miembros de la Mesa, los Grupos Parlamentarios y el Gabinete de Prensa.

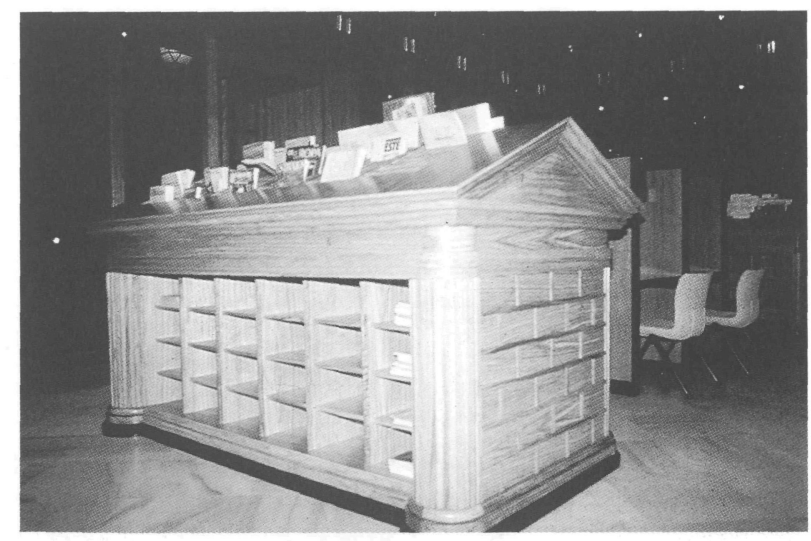

Biblioteca. Detalle de un revistero. (Foto: P. Rodriguez, 1993).
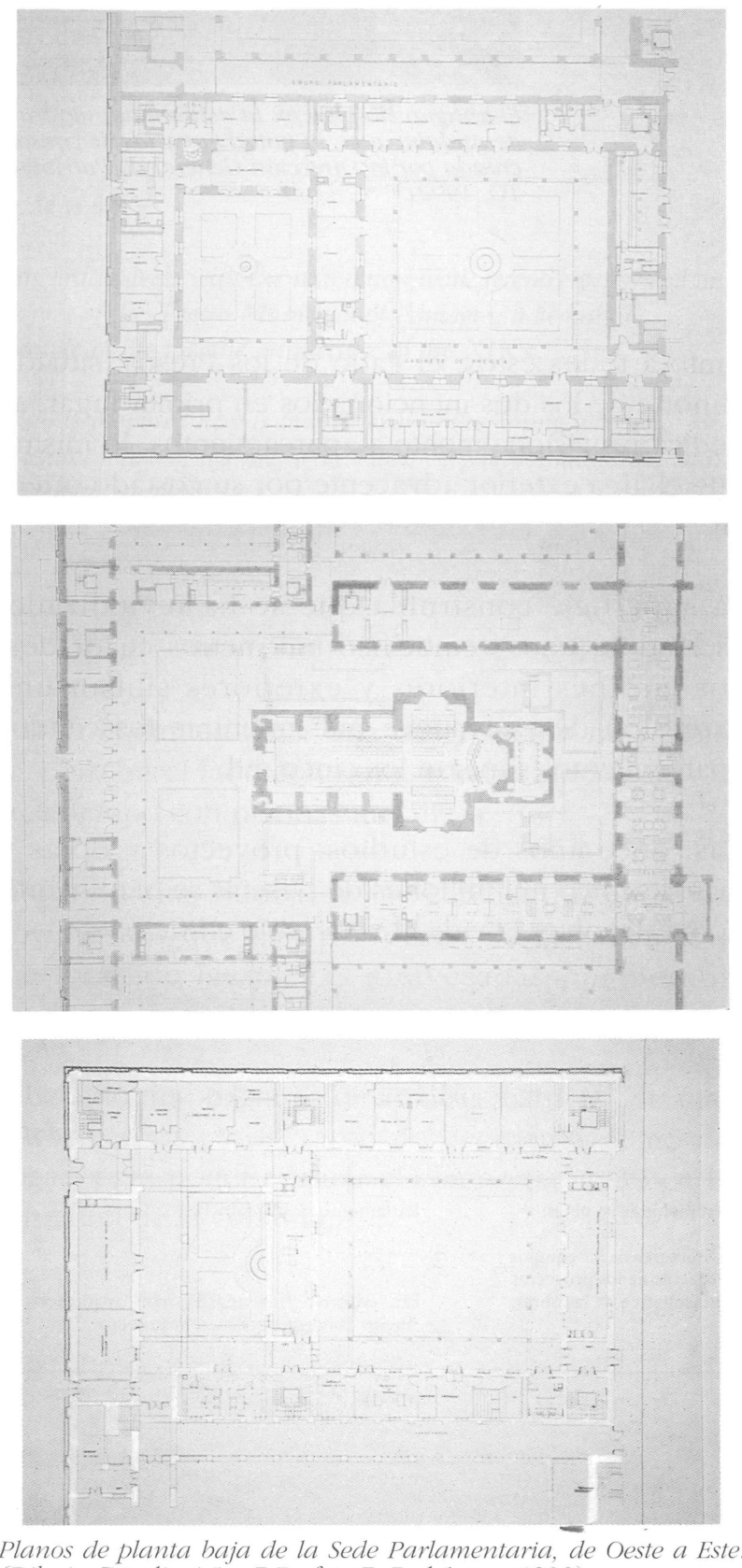

(Dibujo: Estudio A.J. y P.R.; foto P. Rodríguez, 1992) 


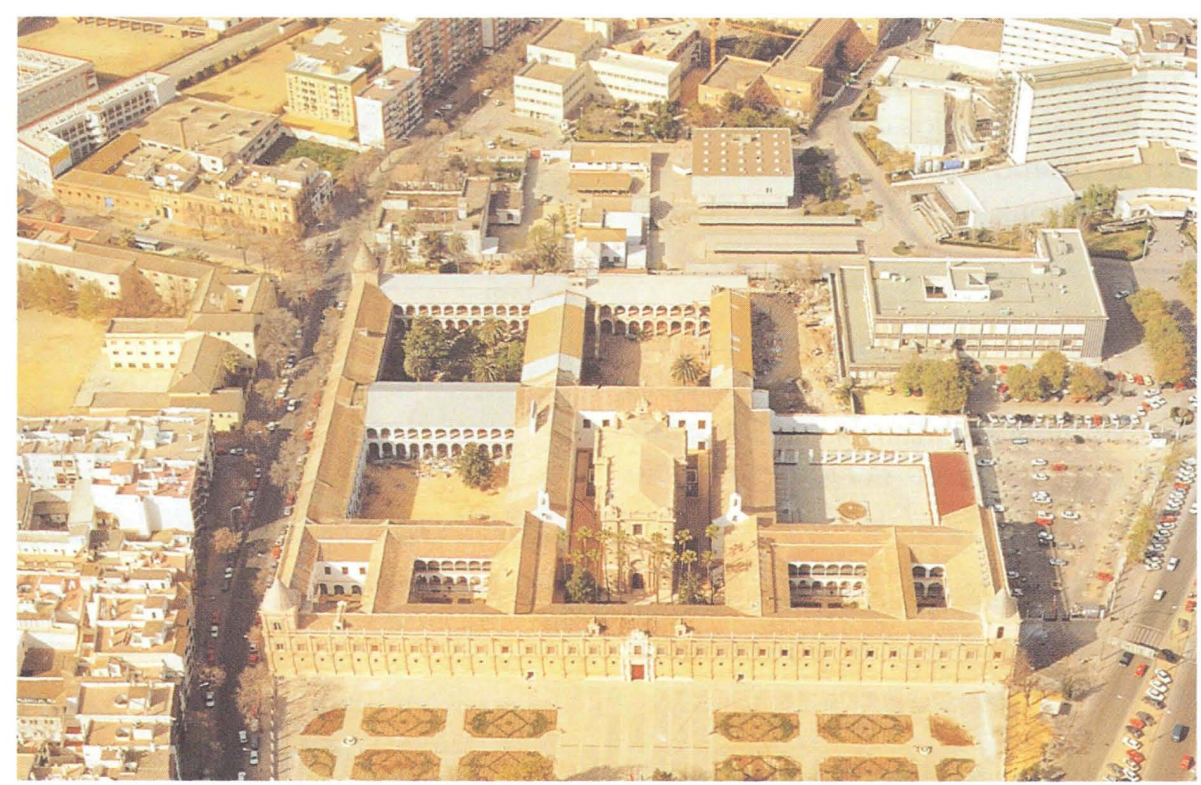

El antiguo Hospital en la actualidad, mostrando las intervenciones promovidas por el Parlamento de Andalucía en la mitad Sur y las de consolidación provisional y limpieza de la zona Norte, ejecutada por la Dirección General de Patrimonio de la Junta de Andalucía. (Foto aérea: TECNIFOTO, 1992).

Junto a todos éstos, el Patio de los Presos, situado al norte de los dos mencionados en primer lugar, se dedica provisionalmente a aparcamientos, lo mismo que el área exterior adyacente por su costado oriental.

La superficie construida que se ha revitalizado, asciende a unos veinticinco mil metros cuadrados. Los jardines interiores y exteriores suman una extensión de casi nueve mil, mientras los citados aparcamientos superan los cinco mil.

Casi once años de estudios, proyectos y obras y más de cinco mil millones de pesetas se han invertido en devolver la vida al venerable edificio.
A pesar de ello, el Hospital de la Sangre, no puede considerarse completamente recuperado, pues su mitad norte todavía permanece desocupada, y se mantiene precariamente en pie gracias a las obras de limpieza, demolición de añadidos y consolidación provisional recién ejecutadas por la Dirección General de Patrimonio de la Junta de Andalucía.

Creemos sinceramente que la categoría del monumento y la dignidad del Parlamento de Andalucía, que lo ha convertido de forma ejemplar en su casa, reclaman la culminación sin demoras de la ingente tarea emprendida.

Que todos la veamos.

\section{Ficha técnica}

\begin{tabular}{|llll}
\hline Promotor de las obras: & Parlamento de Andalucía. & Fecha de los proyectos: & Diciembre de 1982 a diciembre de 1991. \\
$\begin{array}{l}\text { Directores de los equipos } \\
\text { redactor de los proyectos } \\
\text { y facultativo de las obras: }\end{array}$ & $\begin{array}{l}\text { Dr. Alfonso Jiménez Martín, arquitecto y } \\
\text { Pedro Rodriguez Pérez, arquitecto. }\end{array}$ & Fecha de las obras: & Mayo de 1987 a febrero de 1992. \\
\hline
\end{tabular}

